\title{
Teaching Anatomy in Physical Education: Deductive Versus Inductive Methodology
}

\author{
Shahin Ahmedov*, Figen Yaman Lesinger, Onur Sanivar, Perihan Sanivar \\ Cyprus Health \& Social Sciences University, Guzelyurt, N.Cyprus, 99800/via Mersin 10, Turkey
}

Received October 20, 2020; Revised May 7, 2021; Accepted May 16, 2021

\begin{abstract}
Cite This Paper in the following Citation Styles
(a): [1] Shahin Ahmedov, Figen Yaman Lesinger, Onur Sanivar, Perihan Sanivar, "Teaching Anatomy in Physical Education: Deductive Versus Inductive Methodology," International Journal of Human Movement and Sports Sciences, Vol. 9, No. 4, pp. 676 - 681, 2021. DOI: 10.13189/saj.2021.090411.
\end{abstract}

(b): Shahin Ahmedov, Figen Yaman Lesinger, Onur Sanivar, Perihan Sanivar (2021). Teaching Anatomy in Physical Education: Deductive Versus Inductive Methodology. International Journal of Human Movement and Sports Sciences, 9(4), 676 - 681. DOI: 10.13189/saj.2021.090411.

Copyright $\bigcirc 2021$ by authors, all rights reserved. Authors agree that this article remains permanently open access under the terms of the Creative Commons Attribution License 4.0 International License

\begin{abstract}
Substantial knowledge in human anatomy is the premise for constructing lifelong effective exercise programs in public health. The level of public awareness of the basic structure of the human body remains low for years and continues to be a challenge for trainees. The traditional teaching approach in anatomy studies utilizes inductive methodology, where body parts of the structure are taught separately, which might be productive for medical students. Teaching anatomy in nonmedical programs like physical education (PE), however, intends for a holistic understanding of the subject to accentuate functional anatomy, which differs from its classical medical programs purely structural comprehension. Consequently, we suggested that a deductive rather than an inductive form of teaching anatomy in PE might bring the difference and ease the studying process. To statistically analyze this hypothesis traditionally utilized in the PE inductive educational approach for anatomy study was compared with the newly introduced deductive form of teaching through retrospective evaluation of student's exam results. Exam scores of 1167 first-year students were divided into population and sample groups. The former included 1055 exam results of students previously taught by inductive methodology, whereas the latter consisted of 114 students' exam results after the deductive presentation of anatomy. The comparative analysis made by t-test for population-sample groups demonstrated significantly higher $(p<0,001)$ exam scores in the sample group $(40.1 \pm$ 14.2 [95\%CI 37.45-42.73]) comparing to the population
\end{abstract}

group $(34.5 \pm 14.0$ [95\%CI 33.75-35.43]). The limited time of anatomy study and preliminary unawareness of human anatomy made the teacher-centered deductive approach in PE more effective.

Keywords Anatomy Study, Deductive, Inductive, Methodology

\section{Introduction}

Physical activity was reported to reduce ... many chronic diseases and decrease the relative risk for death in the general population $[2,3,4,5,6]$. One crucial way to adapt this behavioral pattern for life is lifelong physical education [8]. Substantial knowledge in human anatomy is the premise both for the general population to understand the importance of physical activity for health and instructors in physical education (PE) to construct lifelong effective exercise programs. The level of public awareness of the basic structure of the human body remains low for years and continues to be a challenge for trainees [7,12]. The traditional process of teaching anatomy in medical school, which counts for hundreds of years [12], provides medical students with essential information about the structure of the human body and creates the foundation for critical reasoning in modern medicine. The conventional teaching of anatomy in 
medical schools utilizes inductive methodology, where body parts are taught separately. Then students gather received information to create the whole picture of the body structure. This approach looks beneficial for medical students since the process continuously underlines the morphological connection of separately learned body parts but can be less advantageous for nonmedical anatomy programs. Due to growing public interest in health, anatomy knowledge has gone beyond the scope of medical school compelled today in various health-oriented programs, including bioengineering, dietetics, and physical education. The ability to create the whole picture of the working body from pieces of information obtained from an inductive form of presentation is often challenging in physical education when students do not have access to cadaver-based instruction and medical imaging technologies so far. Teaching anatomy in PE intends for a holistic understanding of the human body to accentuate functional anatomy, which differs from its classical purely structural comprehension. This alternative educational approach considers separate body parts deducted from general principles of body composition and function. The deductive methodology utilizes known theory/rule, e.g., the safety and ergonomics of posture and movement, to test if this concept is valid for examined findings [1], i.e., alignment and function of the locomotor system, which allows students to comprehend the bodily response to external and internal stimuli. The ability to "read body language" will favor PE students to acquire critical thinking and facilitate the decision-making process. So, the purpose of the study is to define if the deductive approach for human anatomy presentation is more beneficial for PE students comparing to conventionally employed inductive methodology (Figure 1).

\section{Methodology}

\subsection{Study Design}

The exam results of the human anatomy and kinesiology (HAK) course, delivered for 1169 first-year students at the Faculty of Sports Sciences of the Near East University in Nicosia, north Cyprus in 2007-2013 years, were ... included in this randomized controlled study. The lesson was held for 15 weeks with the weekly 3-hour course ( 2 hours of theory +1 hour of practice). The total number of hours for the semester was $15 \times 3=45 \mathrm{~h}$. Anatomy lessons were held in the first half of the week in the morning time, and before physical education classes (gymnastics, athletics). All students were divided into two groups, control and experimental. Those who received conventional (inductive) HAK education between 2007 2012 academic years comprised the control group $(\mathrm{n}=1055)$. The intervention group included 114 students, whom in the 2013 academic year HAK was delivered based on alternatively utilized deductive methodology. There were no ages/gender differences between groups. The subject of education was studying the musculoskeletal system, i.e., one of the essential topics of anatomy study in physical education.

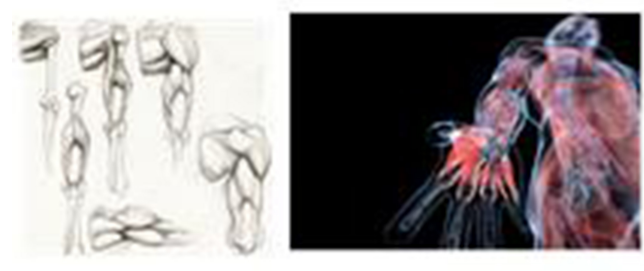

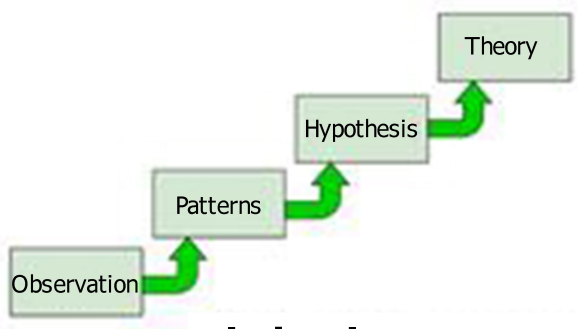

inductive approach
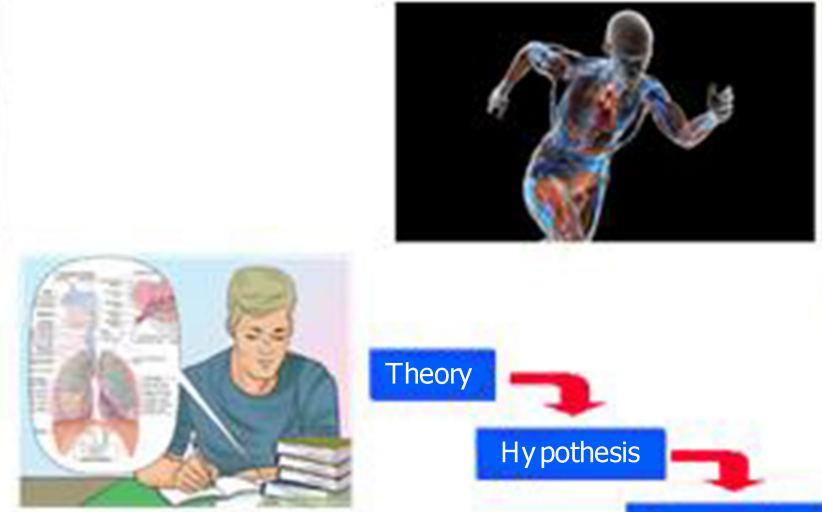

Theory

Hypothesis Observation

Figure 1. Differences between inductive and deductive reasoning in human anatomy studies. 


\subsection{Inductive Methodology}

The inductive educational approach in the control group included the following conventional order of presentation:

A. Skeletal system

1. Upper extremity bones

2. Lower extremity bones

3. Axial skeleton

B. Muscular system

1. Upper extremity muscles

2. Lower extremity muscles

3. Axial skeletal muscles

C. Kinesiology of major joint movements

1. Anatomic planes and axes of movement

2. Shoulder girdle, shoulder joint, elbow, wrist, hand movement patterns

3. Sacroiliac joint, hip, knee, ankle, foot movement patterns

4. Spinal column movement patterns

5. Types of muscle contraction, order of muscle participation in movement, levers, kinetic chains

Students studied separate bones and muscles, after which they tried to apply received information in kinesiology to understand their interaction during joint movement.

\subsection{Deductive Methodology}

The deductive approach, applied as an alternative study of the human locomotor system in the intervention group, started from presenting body parts in the light of principles governing the balance of forces on body posture and movement. The format of this paper does not allow presenting the detailed unfolding of the human locomotor system, so the order of the deductive educational approach was as follows.

A. Spinal column. The spine was presented as the primary determinant of human posture and performance, which continuously struggles against gravity to keep balance and prevent fall. Spinal flexion, i.e., the primary spinal curve in the womb, turns after birth and growth into extension, i.e., secondary for the spinal column curve, to keep upright position against gravity. Location of the abdominal cavity, rib cage, and most of the cranium in front of the spinal column places the latter in the most unfavorable position and aggravates its trend towards flexion. Consequently, we can observe more spinal column extensors in addressing torso muscles compared to the fewer flexor muscles.

B. Upper extremity. The presentation started from the more extensive range of motion in the upper extremity, necessary for daily living activities, developed from its mainly muscular connection with the torso, where the arm connected to the body through the scapula. Thus, the correct scapular position is an essential element of stability in the chain of upper extremity movement. Consequently, those muscles, originating from the spinal column/rib cage and inserting into the scapula, are of paramount importance to attain scapular stability for high performance and injury prevention, particularly in ... overhead sports activities. The working pattern of the upper extremity, mainly in the open kinetic chain, leads to lesser control over the direction of movement but creates greater concentration over separate joints or muscles.

C. Lower extremity. It was underlined that movement of this body part occurred in conjunction with the lumbar spine to keep upright posture and balance. Thus, the connection of the lower extremity to the spine through the pelvic area is more rigid than the upper extremity. Since the lower extremity is the continuation of the spinal column, the hip joint, and lumbar spine share muscles and movements. Muscles of this area often engaged in the movement of two joints, which on many occasions allows corresponding joint interaction during movement. The mainly closed kinetic chain of movement pattern in the lower extremity contributes to the strict ... movement direction and muscle work in groups.

After this introductory presentation, ... each bone/muscle location and function were considered concerning mentioned body tasks for effective and safe postural and movement patterns.

\subsection{Statistical Analysis}

The multiple-choice question type of exams consisted of 30 questions with four possible answer options for each question was adopted for students' performance evaluation. The average exam score of the seven consecutive years $(2007$ - 2012), handled as population score, was evaluated against the average exam score of the 2013 academic year addressing sample score. The t-test for the difference analysis between population and sample means was applied [11]:

$$
\mathrm{t}=\left(\mathrm{X}-\mathrm{M}_{\mathrm{o}}\right) /\left(\mathrm{s}_{\mathrm{x}} / \sqrt{\mathrm{n}_{\mathrm{x}}}\right)
$$

where $\mathrm{X}$ - average sample score, $\mathrm{M}_{\mathrm{o}}$ - average population score, $s_{x}-$ sample score standard deviation, $n_{x}-$ sample size. The statistical threshold for significance of the difference was accepted as $\mathrm{p}<0.05$.

\section{Findings}

Analysis of average exam scores demonstrated a statistically significant difference between deductive and inductive presentations of functional anatomy $(\mathrm{p}<0.001)$. The average \pm standard deviation exam result since 2007 
- 2012 academic years, where the conventional inductive educational approach was applied, was $34.5 \pm 14.0$ (95\%CI, 33.66-35.34), whereas the exam result after the deductive educational approach in the intervention group comprised $40.1 \pm 14.2$ (95\%CI, 37.47-42.73). So, statistical analysis demonstrated that the number of correct answers was higher in the intervention group than in the controls (Figure 2).

\section{Conclusions}

Studies demonstrated a positive correlation between lifelong learning activities and well-being [13]. The growing concern of the population to a healthy lifestyle modality and physical activity, in particular, sets the educational task of preparing qualified PE instructors who would better understand the logic of body structure and function and benefits the decision-making process for exercise programs.

Our observation demonstrated that deductive methodology for human anatomy presentation in freshman PE students facilitated their comprehension of the complexity of functional musculoskeletal anatomy, which improved their exam performance by $16 \%$ (from

\section{$34.5 \pm 14.0$ to $40.1 \pm 14,2$ ).}

During processing any information, we use two main types of thought processes, deduction, and induction. The deduction is the standard way to communicate with students through logic. A teacher explains the rule, decorated with examples, and then a student acquires the subject based on these rules. A deductive methodology is an easiest and fastest way for teachers to convey the functional structure of the human body. The musculoskeletal system, for example, is the unity from functional points of view since they grouped in a logical way to share the same function: to move the body [14]. Since the logic of the rule is at the forefront here, a student who successfully passes the exam, on the other hand, might have a problem with its application in practice. This way, the knowledge gained turns out to be superficial, not persistent, and suitable mainly for typical situations. However, life often becomes more voluminous and broader than the framework, like assessing patients with several overlapping problems. So a person who learned in this way might be lost in any informal situations. Consequently, more time the student should spend on the application of delivered information in the laboratory setting.

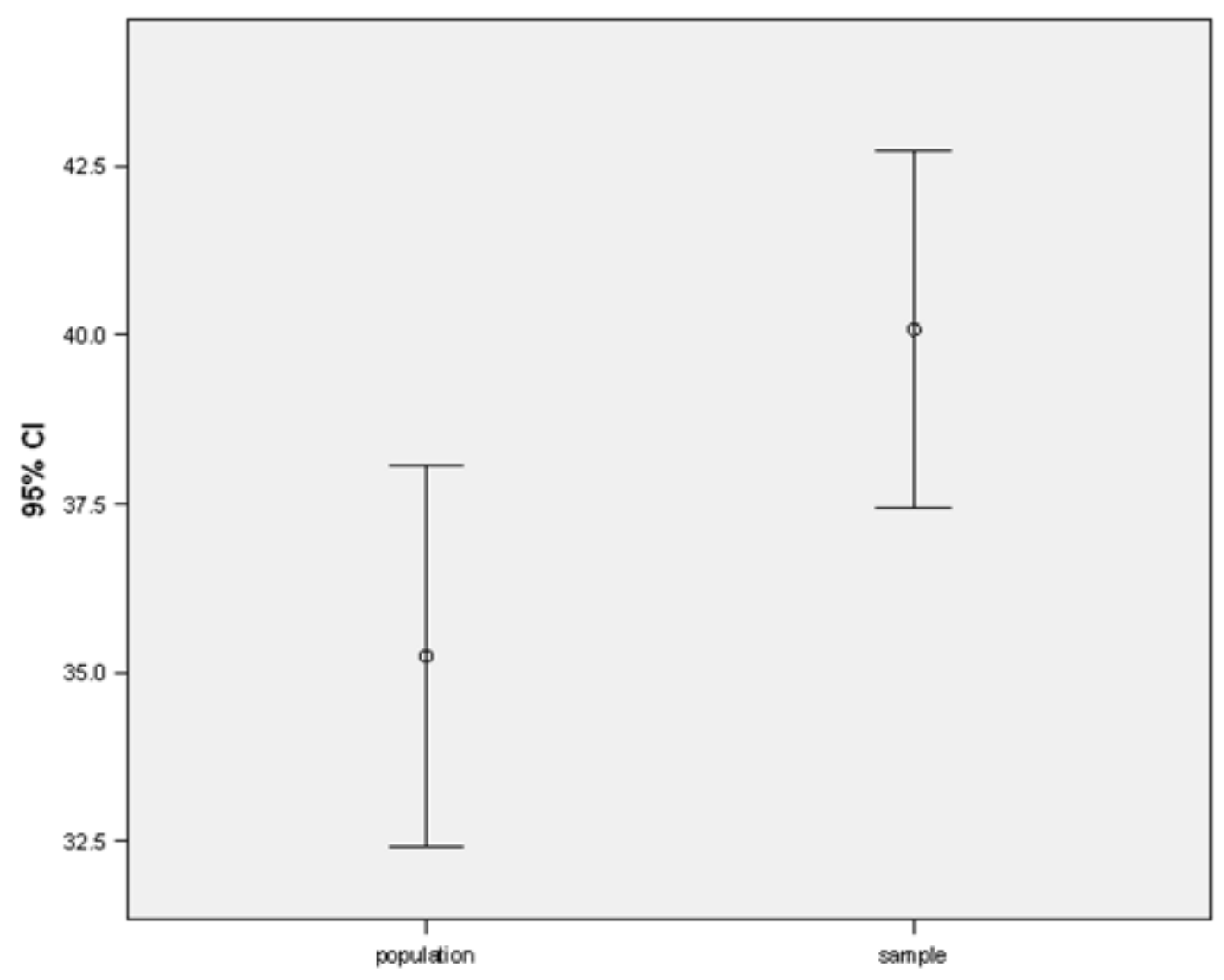

Figure 2. Better exam score in the sample (experimental, $\mathrm{n}=114$ ) group comparing to the population (control, $\mathrm{n}=1055$ ) group. 
In contrast to deduction, induction is an analytical approach (while deduction is synthetic). The student has to observe, analyze in detail separate parts of a subject, and then derive rules of their function, interaction based on the observation and analysis.

Research in ... foreign language teaching has shown that material introduced with active student participation is better comprehended than with passive participation [15]. The student-centered analytical approach is suitable for learning a foreign language, where induction is first included in speech forms and models that the listener assimilates. Active student's participation by using the structure through practice in conjunction with native language, finding similarities and differences, creates patterns and brings questions. So, here the rule, i.e., grammatical explanation, comes to the rescue. This approach is about being able to summarize and make inferences on his own.

Unfortunately, on many occasions, students are not accustomed or have already lost the habit of an active way of acquiring knowledge and want to receive information faster and more specifically, as in an Internet search engine. The human anatomy topic is the subject of biology, taught in educational high school programs. However, ... most of our freshman students came from rural areas, where high school biology programs are mainly agriculture-oriented; their knowledge in human biology might not be well enough to tolerate inductive methodology in undergraduate anatomy study. Besides that, most teachers have many additional formal responsibilities and restrictions. The modern educational format leaves no space or time for creativity and reflection.

The preference for "multiple choice" to "essay" evaluation tool in both groups could be an additional argument for better results in the intervention group. The logical structure of the "multiple choice" form of the examination system requires students to use definitions and assumptions to find the correct answer options, which carry elements of deductive reasoning. "Essay" type of assessment necessitates the use of cognitive skills and creativity to answer exam questions, which might always be challenging for students with a limited preliminary acquaintance with the subject under the question.

Harmoniously, induction and deduction, analysis and synthesis, must go hand in hand in all learning because our brain is designed ... so that it uses both processes of thinking. It would be wrong to say that one of these processes is better and the other worse. It is awful when there is no choice, and only one way is imposed.

Posture and movement are personal characteristics that carry information about its condition, and the effectiveness of corrective exercises will depend on how correctly the PE instructor interprets this body language. Thus, understanding body language in many ways resembles a foreign language study, where the inductive-deductive methodology continues to be a relevant issue. Researchers emphasized the multifactorial influence of the teaching methodology on the student's learning process, where maturity, time allotted for education, and prior awareness of the subject determine the choice of teaching methodology [10]. We admit that the winning educational process will be possible with the correct combination of mainly teacher-oriented deductive and student-oriented inductive teaching methods. However, the limited time of anatomy study in the PE Department and the preliminary ignorance of young students on human anatomy and kinesiology make the teacher-centered deductive approach preferable in teaching functional anatomy as the shorter way to achieve the goal [9].

Of course, efficiency is not always an indicator and criterion for success. Interest, love for the learning subject, correlated with personal experience, are the foundation on which the further development of the individual is based. That is why self-education is often more effective and efficient in all senses of these words since it is more accessible, individually directed, and correlated with personal experience by a person's motivation. Indeed, in many respects, the teacher's task is to show the pupil the path along which the latter will go independently. Of course, the teacher's intention, his interest, which is transmitted to the pupils, is also essential. He can work purely formally, stretch the time until the end of the lesson, or create, construct knowledge. Personal interest and involvement of both parties remove the contradiction in the opposition of deductive and inductive approaches.

\section{Recommendations}

The correctness of the teacher's choice in favor of a particular teaching method of functional anatomy in physical education should be decided individually and primarily determined by the students' readiness to acquire presented material and the composition of the studying process. Further research is necessary to clarify the effectiveness of deductive vs. inductive reasoning in lifelong human anatomy education for other non-medical undergraduate health-related programs.

\section{REFERENCES}

[1] Babbie, E. (2011). The Basics of Social Research. Belmont: Wadsworth Cengage Learning.

[2] Blair, S., Brodney, S. (1999). Effects of physical inactivity and obesity on morbidity and mortality: current evidence and research issues. Med Sci Sports Exerc, 31, 646-662.

[3] Blair, S., Kohl, H., Paffenbarger, R., et al. (1989). Physical fitness and all-cause mortality. A prospective study of 
healthy men and women. JAMA, 262, 2395-2401.

[4] Bouchard, C., Shephard, R. (1994). Physical activity fitness and health: the model and key concepts. In: Bouchard C, Shephard RJ, Stephens T, editors. Physical activity fitness and health: International proceedings and consensus statement. Champaign (IL): Human Kinetics, 77-88.

[5] Lee, I., Skerrett, P. (2001). Physical activity and all-cause mortality: What is the dose-response relation? Med Sci Sports Exerc, 33, 459-471.

[6] Paffenbarger, R., Hyde, R., Hsieh, C., et al. (1986). Physical activity, other life-style patterns, cardiovascular disease and longevity. Acta Med Scand Suppl, 711, 85-91.

[7] Papa, V., Vaccarezza, M. (2013). Teaching anatomy in the xxi century: New aspects and pitfalls. TSWJ, http://dx.doi.org/10.1155/2013/310348.

[8] Sallis, J., McKenzie, T. (1991). Physical education's role in public health. Res Q Exerc Sport, 62, 124-137.

[9] Seliger, H. (1975). Inductive method and deductive method in language teaching: A re-examination. IRAL, 13, 1-18.

[10] Takimoto, M. (2008). The effects of deductive and inductive instruction on the development of language learners' pragmatic competence. Mod Lang J, 92, 369-386.

[11] Thomas, J., Nelson, J. (2001). Research Methods in Physical Activity. Champaign (IL): Human Kinetics.

[12] Turney, B. (2007). Anatomy in a modern medical curriculum. Ann R Coll Surg Engl, 89, 104-107.

[13] Weinman, J., Yusuf, G., Berks, R., Rayner, S., Petrie, K. (2009). How accurate is patients' anatomical knowledge: a cross-sectional, questionnaire study of six patient groups and a general public sample. BMC Fam Prac, 10, doi:10.1186/1471-2296-10-43.

[14] Yamashita, T., Lopez, E., Stevens, J., Keene, J. (2017). Types of learning activities and life satisfaction among older adults in urban community-based lifelong learning programs. Act Adapt Aging, 41(3), 239-257.

[15] Johansson, I., Smith, B., Munn, K., etc. (2005). Functional Anatomy: A Taxonomic Proposal. Acta Biotheor, 53(3), 156-166.DOI:10.1007/s10441-005-2525-3

[16] Gower, R., Phillips, D., and Walters, S. (1995). Teaching Practice. A handbook for teachers in training. Chapter 6: Presenting \& practicing language. Macmillan. 